\title{
The role of fibreoptic bronchoscopy in the investigation of pleural effusion
}

\author{
R.W. Heaton and C.M. Roberts* \\ Department of Medicine, Charing Cross and Westminster Medical School, Fulham Palace Road, \\ London W6 8RF, UK.
}

\begin{abstract}
Summary: Published data on the role of fibreoptic bronchoscopy in the investigation of pleural effusions are sparse and conflicting. We have reviewed our experience from November 1980 to December 1986. Thirty-two patients were identified from bronchoscopy records as having undergone the procedure during the evaluation of an effusion. Fibreoptic bronchoscopy was diagnostic in 6 of the 32 cases, but less invasive procedures had given the diagnosis in 4 , and in the other 2 cases radiological abnormalities other than the effusion suggested an underlying bronchial malignancy. We conclude that routine fibreoptic bronchoscopy is not justified in the evaluation of pleural effusions.
\end{abstract}

\section{Introduction}

The underlying cause of a pleural effusion may be readily apparent from the clinical features of the case, but on occasion diagnosis can be difficult and require intensive evaluation.

The role of fibreoptic bronchoscopy (FOB) in the investigation of patients who present with a pleural effusion is not well defined. Although the inclusion of bronchoscopy as part of the routine evaluation of all such patients is frequently advocated, ${ }^{1}$ evidence to support this is sparse. Williams \& Thomas $^{2}$ obtained a diagnosis in only 4 of 28 patients bronchoscoped because of unexplained pleural effusion. Of these, three patients found to have a bronchial carcinoma were cigarette smokers with haemoptysis - a definite indication for bronchoscopy in itself. ${ }^{3}$ In a larger series, excluding all patients with other indications for bronchoscopy, Feinsilver et al. ${ }^{4}$ were unable to support the routine use of FOB.

In an attempt to clarify the role of FOB in the investigation of pleural effusion we have reviewed the records of 32 patients who presented with a pleural effusion and inderwent FOB.

\section{Patients, methods and results}

FOB records of Charing Cross Hospital from November 1980 to December 1986 were searched to identify patients undergoing the procedure for the investigation of a pleural effusion. Thirty-two

Correspondence: R.W. Heaton, M.R.C.P.

*Trustees Research Fellow

Accepted: 13 January 1988 patients (20 male; mean age 63.5 years; 26 smokers) were identified. Case notes and $\mathrm{X}$-rays were reviewed to obtain details of investigations and final outcome. Pleural fluid was examined microbiologically and cytologically in all. Closed pleural biopsies were performed in 25. FOB was carried out under local anaesthetic and samples obtained for microbiology, cytology, and, where indicated, histology.

In 14 of the 32 patients pleural effusion was the only indication for FOB. In 18 patients a separate radiological abnormality or haemoptysis provided a standard indication for the procedure. Diagnoses were made in 30 (Table I). In 6 cases FOB provided a definite diagnosis. Four of these (carcinoma 3, tuberculosis 1) were confirmatory of results obtained from less invasive investigations. Both cases where FOB alone gave a diagnosis were of bronchial carcinoma, and, in both, radiological appearances other than the effusion indicated an

Table I Final diagnoses in 30 patients

\begin{tabular}{lll} 
Carcinoma & & 14 \\
$\quad$ Primary bronchial & 9 & \\
$\quad$ Secondary, identified source & 2 & \\
$\quad$ Unknown primary & 3 & \\
Empyema/parapneumonia & 4 \\
Mesothelioma & 3 \\
Tuberculosis & 2 \\
Asbestos pleural disease & 2 \\
Congestive cardiac failure & 2 \\
Chronic renal failure + infection & 1 \\
Pulmonary embolus & 1 \\
Lymphoma & 1 \\
\hline
\end{tabular}

(C) The Fellowship of Postgraduate Medicine, 1988 
underlying carcinoma. Of the 14 cases with otherwise normal chest $\mathrm{X}$-rays, endobronchial malignancy was found in two, both of whom had positive pleural fluid cytology. Diagnostic results were obtained on simple aspiration and closed pleural biopsy in 12 of the 32 cases, with open biopsy adding five more diagnoses.

\section{Discussion}

This retrospective survey does not support the routine use of FOB in the investigation of patients with pleural effusion. In the two cases where FOB provided a diagnosis that had not been obtained

\section{References}

1. Light, R.W. In: Pleural Disease. Lea and Febiger, Philadelphia, 1983, pp. 56-57.

2. Williams, T. \& Thomas, P. The diagnosis of pleural effusions by fibreoptic bronchoscopy and pleuroscopy. Chest 1981, 80: 566-569.

3. Santiago, S.M., Lehrman, S. \& Williams, A.J. Bronchoscopy in patients with haemoptysis and normal chest roentgenograms. $\mathrm{Br} J$ Dis Chest 1987, 81: 186-188. from less invasive procedures, other radiological abnormalities would have indicated the need for bronchoscopy. Localizing a bronchial primary in patients with a malignant pleural effusion is unlikely to influence patient management or outcome. ${ }^{5}$ Our results agree with those of Feinsilver et al. ${ }^{4}$ Although Williams \& Thomas $^{2}$ concluded differently, we would suggest their results also support the view that FOB should not be included routinely in the investigation of pleural effusion. Initial investigations should be by pleural aspiration and biopsy. FOB should be performed only in those patients who have independent clinical evidence suggestive of a bronchial carcinoma.

4. Feinsilver, S.H., Barrows, A.A. \& Braman, S.S Fibreoptic bronchoscopy and pleural effusion of unknown origin. Chest 1986, 90: 516-519.

5. Kirsten, F., Chi, C.H., Leary, J.A., Ng, A.B.P., Hedley, D.W. \& Tattersall, M.H.M. Metastatic adeno or undifferentiated carcinoma from an unknown primary site - Natural history and guidelines for identification of treatable subjects. $Q J \mathrm{Med} 1987,62: 143-161$. 